\title{
Challenges in Management of Epithelioid Hemangioendothelioma of the Spine: The Report of a Rare Cause of Cauda Equina Syndrome
}

\author{
Keyvan Eghbal ${ }^{1}$, Nima Derakhshan ${ }^{1,{ }^{*}}$, Arash Saffarian ${ }^{1}$, Hamed Yazdanpanah ${ }^{1}$ and Amirreza \\ Dehghanian ${ }^{2,3}$ \\ ${ }^{1}$ Neurosurgery Department, Shiraz University of Medical Sciences, Shiraz, Iran \\ ${ }^{2}$ Trauma Research Center, Shiraz University of Medical Sciences, Shiraz, Iran \\ ${ }^{3}$ Molecular Pathology and Cytogenetic Ward, Department of Pathology, Shiraz University of Medical Sciences, Shiraz, Iran \\ "Corresponding author: Neurosurgery Department, Shiraz University of Medical Sciences, Shiraz, Iran. Email: nderakhshan26@gmail.com
}

Received 2018 October 10; Accepted 2019 September 18.

\begin{abstract}
Introduction: Epitheliod hemangioendothelioma (EHE) is a rare spinal neoplasm with vascular provenance, which can present as a primary lesion or metastatic lesion with the secondary involvement of the liver, lung, and long bones. EHE can occur all over the spine from the craniovertebral junction to the lumbosacral area. Usual presenting symptoms are back pain, pathologic fractures, myelopathy, and cauda equina syndrome. Diagnosis and management can be difficult, often requiring coordination between multiple disciplines for selective spinal angiography, surgical resection, the establishment of pathologic diagnosis, and adjuvant treatment, if necessary. There are no widely accepted guidelines for the management of EHE.

Case Presentation: We report a 43-year-old male as a case of primary unifocal hemangioendothelioma with two unique features compared to previous reports: first, the presentation with acute cauda equine syndrome and second, the involvement of posterior elements, which made it very difficult to differentiate from aneurysmal bone cyst (ABC).

Conclusions: We review the existing literature on spinal EHE, present management challenges via case review, and propose a management guideline for neurosurgeons, interventional radiologists, pathologists, and radiation oncologists to streamline the diagnosis and management of EHE of the spine.
\end{abstract}

Keywords: Guideline, Epitheoid Hemangioendothelioma, Spine

\section{Introduction}

Epithelioid hemangioendothelioma (EHE) is a rare neoplasm of vascular provenance, first described by Weiss and Enzinger in 1982 (1), as an abnormal growth of endothelial cells with "epithelioid" and "histiocytoid" features, which was commonly mistaken for carcinomas. These histologic features are within a spectrum between epithelioid hemangioma and epithelioid angiosarcoma (2), which are its benign and malignant counterparts, respectively. However, with the help of immunohistochemistry (IHC), the establishment of a definitive pathologic diagnosis of EHE has become easier and more accurate.

The primary sites of involvement include the liver (3, 4), lungs (5-7), bones (8-11), and skin (12). The estimated prevalence of distant metastasis is between 20 and 30\% with varying local recurrence rates from 0 to as high as 13\% (13). EHE accounts for less than $1 \%$ of osseous tumors (14) and the involvement of the vertebral column is even rarer (less than $10 \%$ of osseous EHEs) (14). Spinal involvement may be primary or secondary to metastases from a distant source. Thoracic spine (13, 15, $16)$ is the most common site of involvement, followed by cervical (13, 17-19), lumbosacral (8, 20-23), and craniovertebral junction (24). Diagnosis is usually made by imaging studies showing an osteolytic lesion, with the involvement of the vertebral body. However, the involvement of the dorsal spine has also been reported, which makes the diagnosis further challenging by mimicking lesions such as aneurysmal bone cysts (ABC).

Although most patients present with neck and back pain, neurological catastrophes may occur following intratumoral hemorrhage, epidural growth, and the collapse of vertebrae, resulting in varying degrees of permanent deficits. 
Given the relative rarity of this lesion, there are no widely accepted guidelines for the management of patients with EHE of the spine. Herein, we aimed to summarize the current literature on spinal EHE and propose a management guideline for simplifying the approach to this rare spine tumor.

\section{Case Presentation}

A 43-year-old male was referred to our Neurospine Department, due to acute-onset both lower extremities weakness and urinary retention for 12 days before admission. Except for low back pain for the last six months (VAS = 8 - 9), his past medical history was unremarkable. On physical examination, he had lumbar tenderness on the midline, muscle power of 3 out of 5 in knee extension, and ankle dorsiflexion of both lower limbs and saddle hypesthesia.

Computed tomography scans revealed an expansile osteolytic lesion in the second lumbar vertebrae with an egg-shell pattern, involving both pedicles and posterior elements (Figure 1). Magnetic resonance imaging showed a lesion at L2 with iso- to hyper-intense signals on T1- and a mixed pattern on T2-weighted images (Figure 2). He also underwent selective spinal angiography a day before surgery, which revealed a tumor blush at L2 (Figure 3) and embolization with polyvinyl alcohol (PVA) was performed. The large bony vascular mass involving the spinous process, laminae, facets, and pedicles of L2 was totally excised along with the curettage of suspicious portions of the L2 body. The cavity in the L2 body was augmented with polymethyl methacrylate (PMMA) cement and posterior spinal fixation from T12 to L4 was carried out (Figure 4).
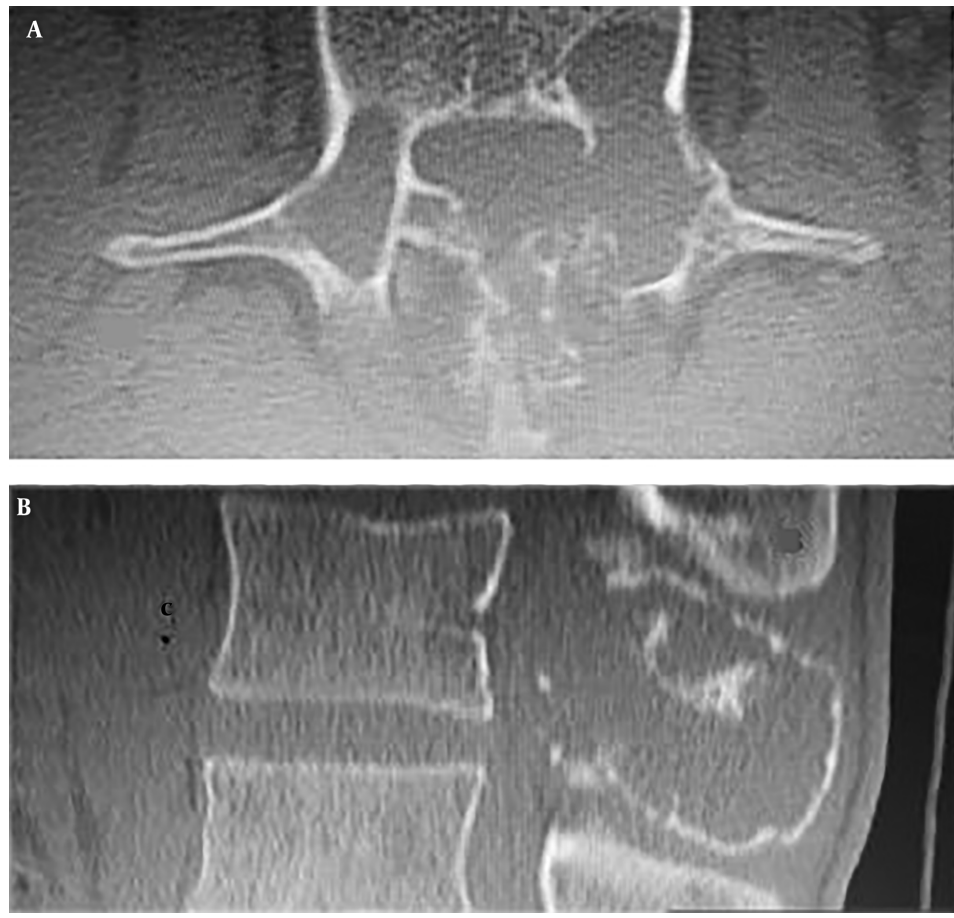

Figure 1. Axial (A) and sagittal (B) computed tomography scans demonstrating an expansile osteolytic lesion in pedicles of L2, with the involvement of the posterior element

Histopathology revealed tumor cells with plasmacytoid features and eccentric round nuclei had made some small spherical spaces between the cells. Immunohistochemical staining showed strong positivity for CD31 and weak cytoplasmic positivity for factor VIII, which made the diagnosis of hemangioendothelioma unmistakable (Figure 5).

In a one-year follow-up, he was pain-free (VAS $=0$ ) and regained normal muscle power of lower extremities, as well as sphincter control. X-ray imaging at the follow-up visit confirmed no recurrence (Figure 6). 


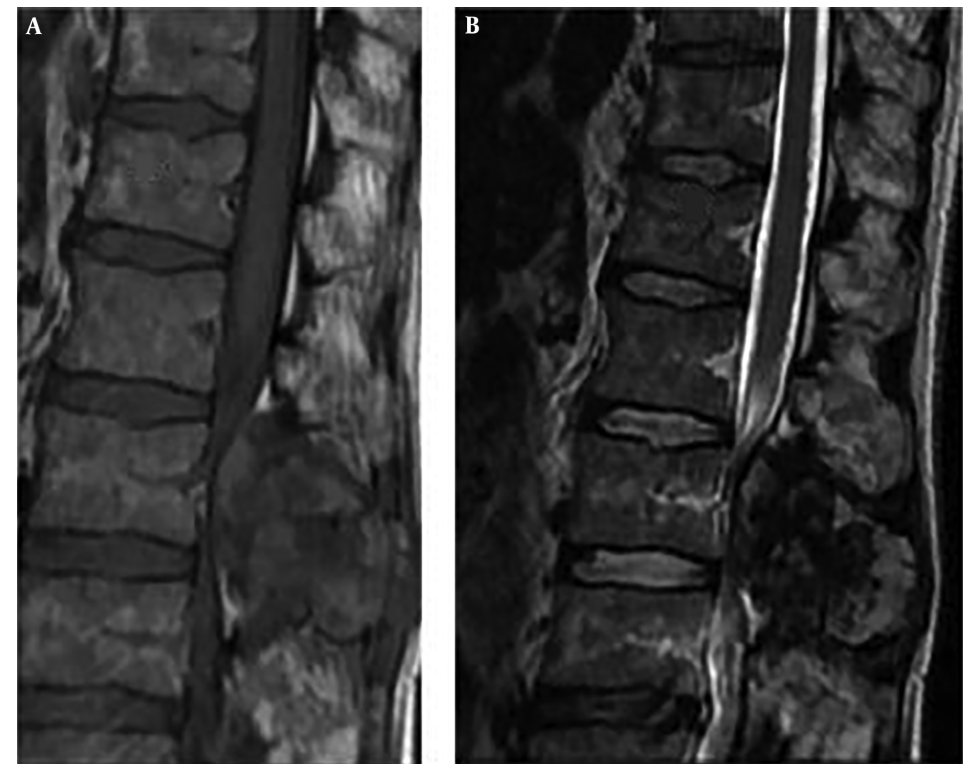

Figure 2. A, Sagittal T1-W MRI showing an iso- to hyper-intense lesion at L2, with compression on cauda equina at this level; B, T2-W MRI showing the lesion with mixed intensities

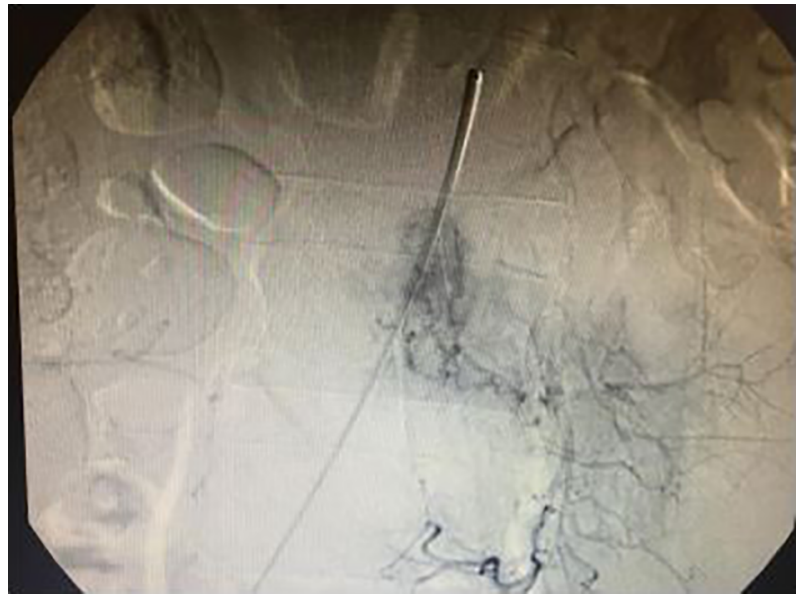

Figure 3. Selective spinal angiography showing a tumor blush at L2

\section{Discussion}

Herein, we provide a literature review of spinal EHE with particular emphasis on clinical presentation, radiologic diagnosis, and treatment.

\subsection{Presentation}

Although most patients present with benign symptoms such as neck and back pain, acute neurologic deterioration is not uncommon. Myelopathy (17) and cauda equina syndrome may result from the epidural compression of tumor growth, as well as pathologic fractures. Even though not reported in the literature, EHE like other vascular neoplasms of the spine such as $\mathrm{ABC}$ and hemangioma has the propensity to cause neurological compromise with epidural hematomas, either 

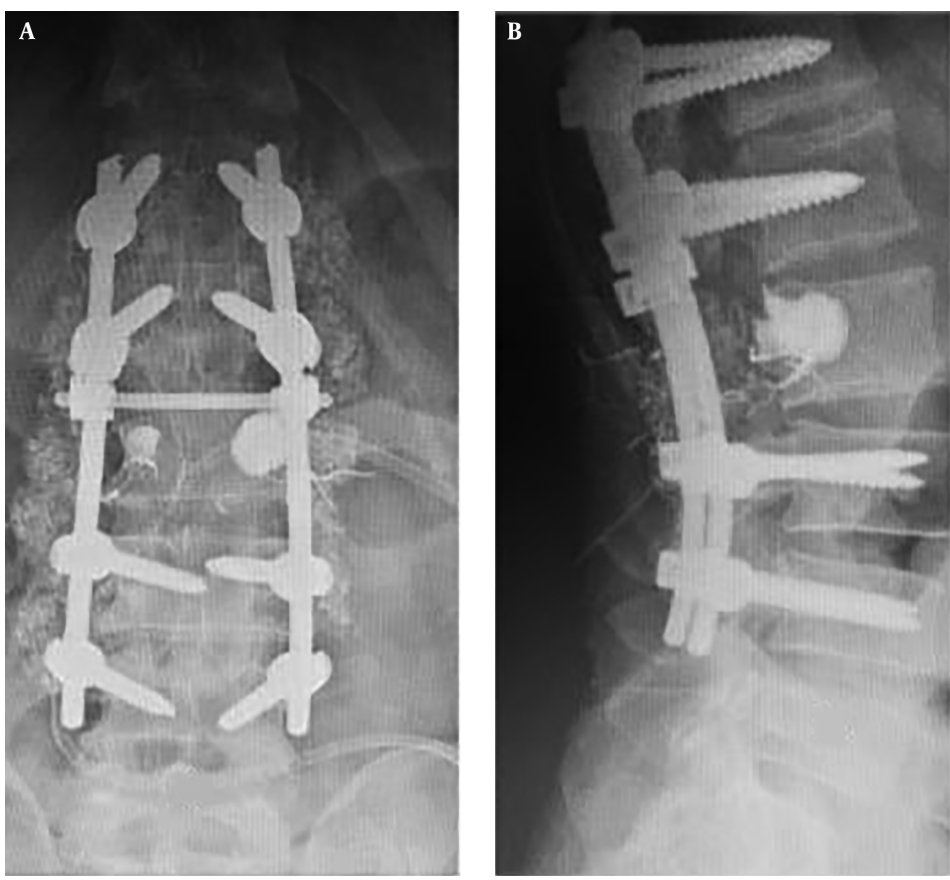

Figure 4. Postoperative X-ray image
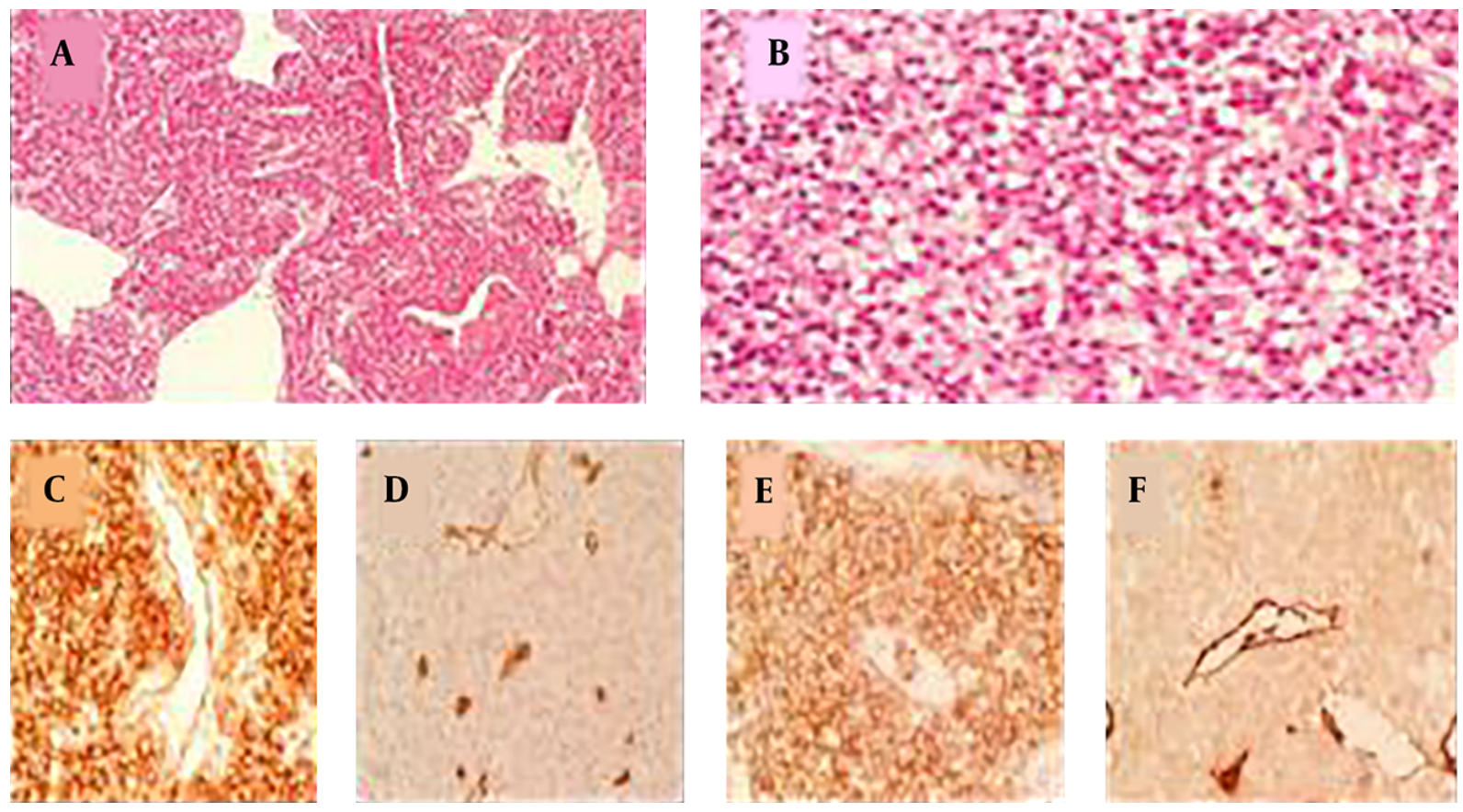

Figure 5. Histopathology and immunohistochemistry of the tumor. A, A sheet of tumor cells with cleft like spaces (H \& E, $40 \times)$; B, tumor cells with plasmacytoid feature and eccentric round nuclei making some small spherical spaces between the cells $(\mathrm{H} \& \mathrm{E}, 400 \times)$; C, immunohistochemical staining shows strong membranous positivity for CD31 $(400 \times)$; D, EMA $(400 \times)$; F, weak cytoplasmic positivity for factor VIII $(400 \times)$; D, negativity for CD34 $(400 \times)$. 

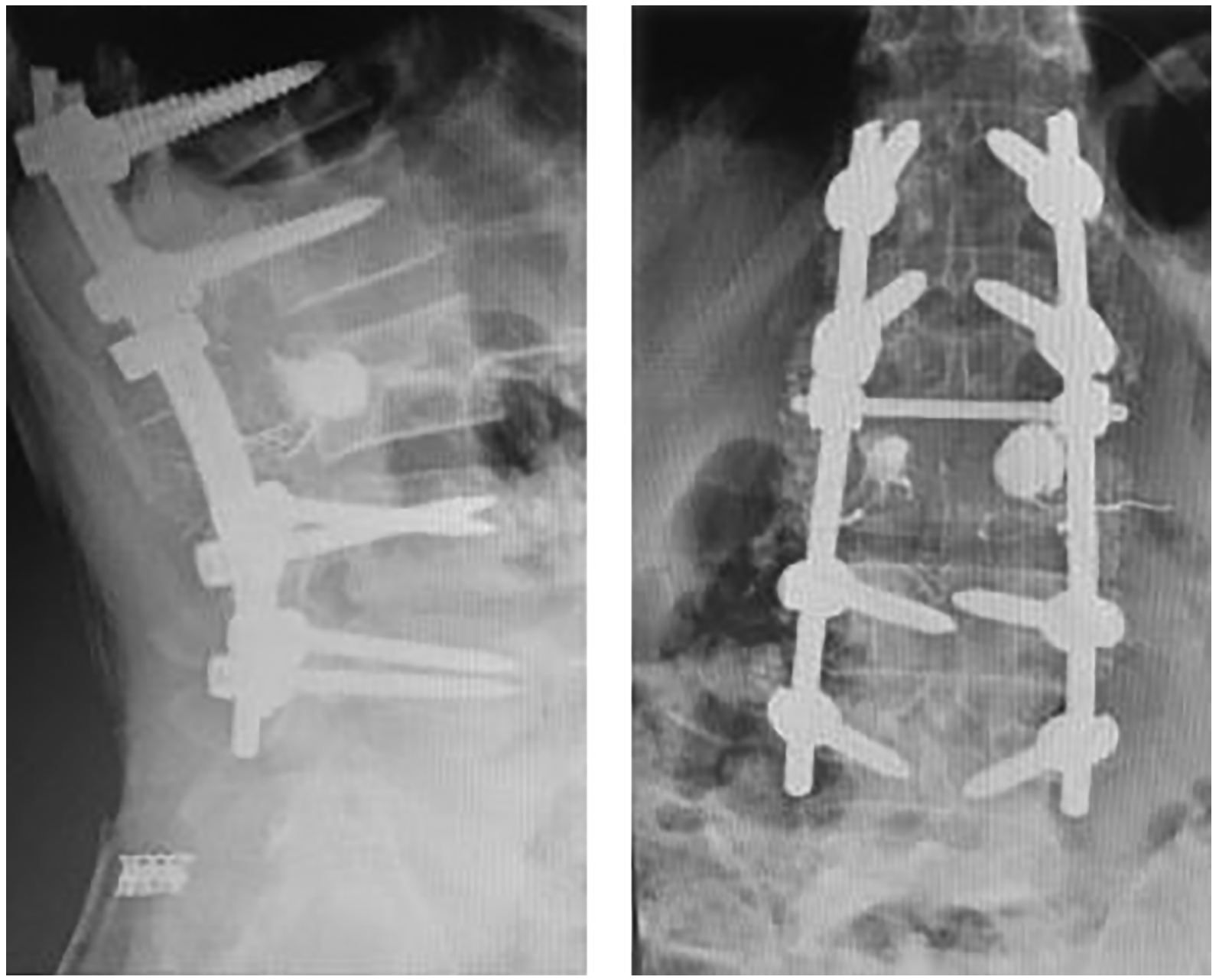

Figure 6. One-year follow-up X-ray showing no recurrence, maintenance of vertebral body height, and balance of spinal column.

spontaneously or following minor traumas. Another potential cause of neurological compromise with EHE may be the vascular steal, particularly in watershed areas such as the lower thoracic spine, which mimics the picture of spinal arteriovenous malformation, as well.

\subsection{Origin and Genetics}

Hemangioendothelioma is derived from endothelial cells, thus rationalizing its presentation in organs such as the liver, broncho-alveolar tissue, skin, and cancellous bones such as vertebrae where they are abundantly found.

Two fusion genes have been described in EHEs; WWTR1-CAMTA1 (due to a reciprocal translocation $\mathrm{t}(1 ; 3)$ (p36;q25)), which is the most common abnormality, and YAP1-TFE3, which has been described in a few remaining cases (25-27).

A correlation between neurofibromatosis type1(NF1) and EHE was also described in a patient harboring multiple dural ectasias, a thalamic/peduncular astrocytoma grade II and thoracolumbar EHE (28).

\subsection{Pathology}

\subsubsection{Histologic Findings}

Common histologic findings include rounded or slightly spindled endothelial cells arranged in strands, cords, and nests. These epithelioid cells have abundant hyaline cytoplasm with prominent cytoplasmic vacuolization, vesicular nu- 
clei, and small distinct nucleoli. Nuclear atypia is usually present.

\subsubsection{IHC Findings}

The positivity of FLI1, ERG, CD31, CD34, and factor VIII-related antigen is seen, which is suggestive of endothelial differentiation. Reactivity for epithelial markers such as cytokeratin and epithelial membrane antigen (EMA) is negative or focally positive.

\subsection{Imaging Signs}

3.4.1. Computed Tomography

Most lesions appear as well-demarcated osteolytic lesions with a thin sclerotic margin (egg-shell pattern) on CT scan. Most lesions involve vertebral bodies, but expansion from pedicles toward dorsal elements is not uncommon (Figure 1).

\subsubsection{MRI Findings}

These lesions appear as iso- to hyper-intense on T1-weighted images and have a mixed pattern on T2-weighted images (23). On T1-weighted images after Gadolinium injection, most lesions show homogenous enhancement.

\subsubsection{Selective Spinal Angiography}

Selective spinal angiography is necessary not only to evaluate the vascular supply of spinal EHE, but also for the consideration of pre-operative embolization to facilitate the subsequent surgical resection by decreasing blood loss.

\subsection{Treatment}

\subsubsection{Indications}

Patients who suffer from lesions, which present with pain and pathologic fractures with the intact neurologic examination and are diagnosed with a biopsy, may be considered for elective staged embolization and resection. Neurological deterioration is an absolute indication for emergency embolization and decompression.

\subsubsection{Standard Treatment Protocol}

Like many other vascular neoplastic lesions of the spine, standard treatment protocol consists of staged preoperative embolization, followed by the complete resection of the lesion and subsequent reconstruction of the vertebrae and stabilization of the spinal column.

Whenever a safe resection is not possible (e.g. proximity to vasculature (13), inoperable sites (29) or multiple sites of involvement), minimally-invasive approaches such as vertebroplasty, cryoablation, and external radiation can be followed.

In case that en bloc resection is feasible, the patient can be followed with serial imaging studies and clinical examination. Otherwise, which is the case of most patients, postoperative conventional radiotherapy is required to ensure that the lesion does not recur.

\subsubsection{Role of Vertebroplasty}

Besides providing the stability and maintenance of the height, the augmentation of vertebrae with PMMA cement is shown to possess oncolytic effects on certain tumors (30). The lack of osseointegration seen with PMMA cement has made calcium phosphate and allograft materials more interesting in this setting, which further incorporates into normal surrounding bones (22).

\subsubsection{Percutaneous Cryoablation}

Sebastian et al. (22) reported on a minimally-invasive approach for percutaneous cryoablation of EHE in the second lumbar vertebrae, considering that their patient had L5-S1 spondylolisthesis that would further complicate posterior fusion surgery in the 18-year-old, neurologically intact patient. They utilized a sequential treatment protocol by transpedicular and lateral paraspinal approaches with CT fluoroscopic guidance for biopsy and cryoablation of the lesion, followed by a transpedicular approach for curettage and deployment of an allograft-filled polyethylene mesh bag on the next day. At a 3.5-year follow-up, the patient had neither pain nor any sign of recurrence and maintained the vertebral body height through the incorporation of allograft with normal L2 body. 


\subsubsection{Radiation}

Both conformal and proton beam radiations can be applied as palliative measures for the treatment of recurrence cases, patients who are not suitable candidates for surgery, and those for whom en bloc resection was not feasible.

A report on 24-month recurrence-free survival of a patient with EHE of the spine, who developed inoperable recurrence using helical TomoTherapy ${ }^{\circledR}$ with intensity modulation, supported the role of external radiation as a palliative measure (29).

\subsection{Follow-up Protocol}

Most authorities agree that follow-up sessions after treatment can be provided at the 1st, 3rd, 6th, and 12th months and yearly thereafter, following surgery to evaluate possible recurrence and initiate the timely management.

\subsection{Lessons from the Case}

This case offers the following lessons that are broadly applicable to many spinal EHEs:

1) In cases with acute neurological deterioration, we should consider the standard treatment protocol with emergency embolization, resection of the tumor, and subsequent reconstruction and stabilization of the spinal column.

2) This case had two unique presentations not previously reported in the literature; the first presentation was cauda equina syndrome and the second one was the involvement of pedicles and posterior elements. According to the threecolumn theory of Denis, the involvement of dorsal and middle Denis columns made his spine unstable, thus justifying the need for posterior spinal fixation along with the resection of the mass and PMMA augmentation.

3) Our primary diagnosis based on imaging findings was ABC. However, EHE should be in consideration when an osteolytic lesion involving the posterior elements primarily is encountered.

\subsection{Management Guideline}

Considering the presented symptoms, the patient's general condition, previous treatment modalities, and availability of therapeutic options, a guideline is proposed to simplify the diagnosis and management of EHEs, as shown in Figure 7.

\subsection{Conclusions}

Hemangioendothelioma should be considered in the differential diagnosis when a primary vascular neoplasm of the spine is encountered. Treatment modalities consist of conservative measures, minimally-invasive procedures, standard corpectomy plus reconstruction, and stabilization and radiation. The choice of therapy should be considered in a multidisciplinary fashion and on an individual basis, taking into account the patient's general condition, degree and level of involvement of vertebrae, the number of levels involved, and the severity of symptoms.

\section{Footnotes}

Conflict of Interests: There is no conflict of interest in this study.

Funding/Support: This article had no source of funding.

Informed Consent: Informed consent was obtained from the patient that is available for the Editorial Office.

\section{References}

1. Weiss SW, Enzinger FM. Epithelioid hemangioendothelioma: A vascular tumor often mistaken for a carcinoma. Cancer. 1982;50(5):970-81. doi: 10.1002/1097-0142(19820901)50:5<970::aid-cncr2820500527>3.0.co;2-z. [PubMed: 7093931].

2. O'Connell JX, Kattapuram SV, Mankin HJ, Bhan AK, Rosenberg AE. Epithelioid hemangioma of bone. A tumor often mistaken for low-grade angiosarcoma or malignant hemangioendothelioma. Am J Surg Pathol.1993;17(6):610-7. [PubMed: 8333560].

3. Mehrabi A, Kashfi A, Fonouni H, Schemmer P, Schmied BM, Hallscheidt P, et al. Primary malignant hepatic epithelioid hemangioendothelioma: A comprehensive review of the literature with emphasis on the surgical therapy. Cancer. 2006;107(9):2108-21. doi: 10.1002/cncr.22225. [PubMed: 17019735].

4. Lauffer JM, Zimmermann A, Krahenbuhl L, Triller J, Baer HU. Epithelioid hemangioendothelioma of the liver. A rare hepatic tumor. Cancer. 1996;78(11):2318-27. doi: 10.1002/(sici)1097-0142(19961201)78:11<2318::aid-cncr8>3.0.c0;2-i. [PubMed: 8941001]. 

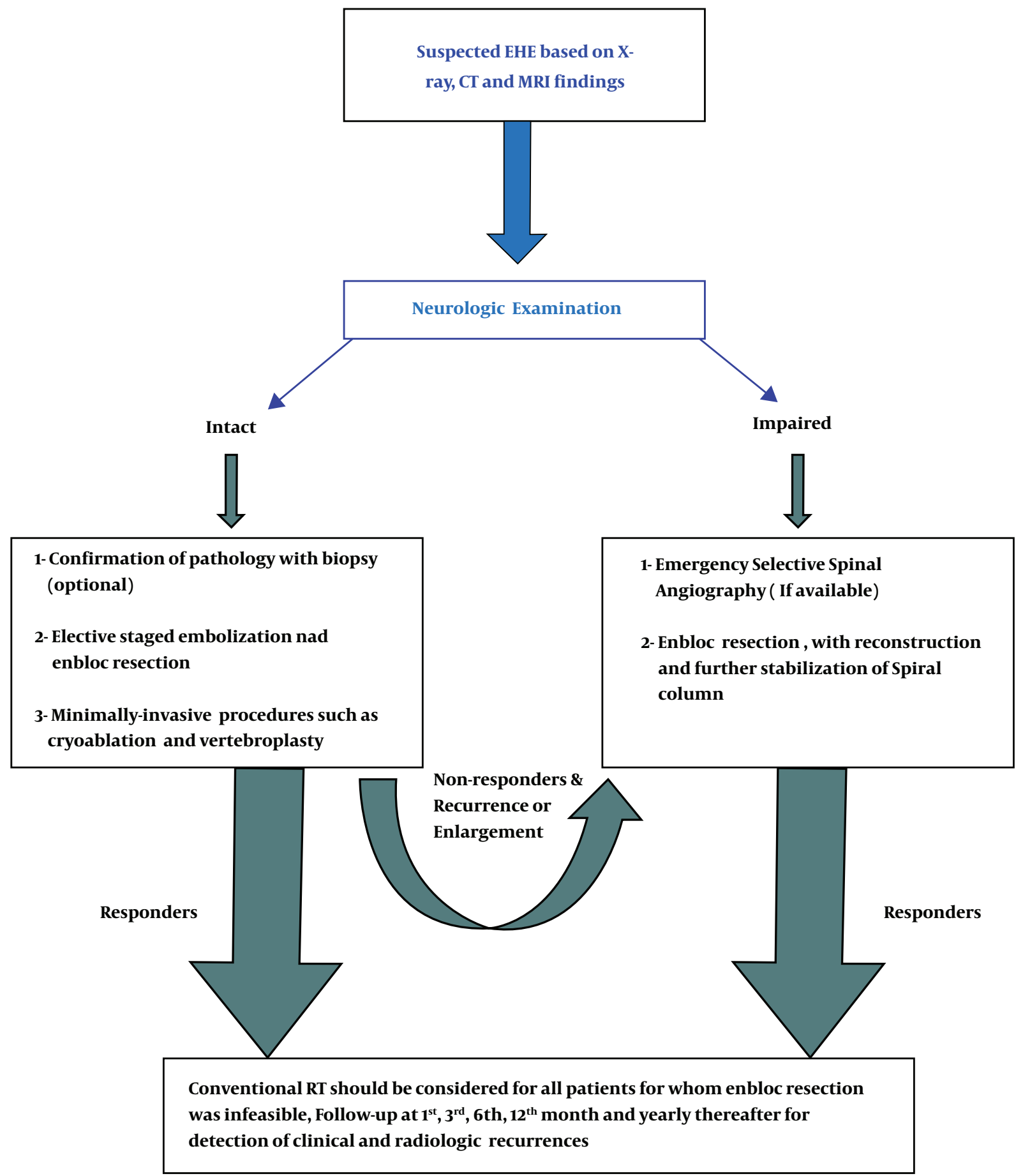

Figure 7. Management guideline for EHE of the spine

5. Kim KY, Kim CH, Sohn JW, Cha SI, Chae SC, Park JY, et al. A case of pulmonary epithelioid hemangioendothelioma. Tuberc Respir Dis. 1999;47(5):691. doi: 10.4046/trd.1999.47.5.691.

6. Rock MJ, Kaufman RA, Lobe TE, Hensley SD, Moss ML. Epithelioid hemangioendothelioma of the lung (intravascular bronchioloalveolar tumor) in a young girl. Pediatr Pulmonol. 1991;11(2):181-6. doi:10.1002/ppul.1950110217. [PubMed: 1661888]. 
7. Arasanz HE, Hernandez BM, Perez SR, Lacalle AE, Perez LR, Martinez MA. [Highly aggresive pulmonary epithelioid hemangioendothelioma: A case report]. An Sist Sanit Navar. 2017;40(2):309-14. Spanish. doi: 10.23938/ASSN.0035. [PubMed: 28765663].

8. Das JM, Louis R, Sunilkumar BS, Peethambaran AK. Primary epithelioid hemangioendothelioma of the dorsal spine: A review. Neurol India. 2017;65(5):1180-6. doi:10.4103/neuroindia.NI_896_16.[PubMed:28879934].

9. Weissferdt A, Moran CA. Epithelioid hemangioendothelioma of the bone: A review and update. Adv Anat Pathol. 2014;21(4):254-9. doi: 10.1097/PAP.0000000000000027. [PubMed: 24911250].

10. Plumby MC, Bacaj P, Lindsey BA. Unicentric epithelioid hemangioendothelioma of the calcaneus: A case report and review of literature. Clin Sarcoma Res. 2018;8:5. doi:10.1186/s13569-018-0092-z. [PubMed: 29632658]. [PubMed Central: PMC5887220].

11. Rasool MN, Snyders RF, Bydawell G. Epithelioid haemangioendothelioma of the scapula in a child: A case report and review of the literature. South Afr Orthop J. 2015;14(4):62-6. doi: 10.17159/2309-8309/2015/v14n4a9.

12. Mentzel T, Beham A, Calonje E, Katenkamp D, Fletcher CD. Epithelioid hemangioendothelioma of skin and soft tissues: Clinicopathologic and immunohistochemical study of 30 cases. Am J Surg Pathol.1997;21(4):363-74. doi: 10.1097/00000478-199704000-00001. [PubMed: 9130982].

13. Aquilina K, Lim C, Kamel MH, Marks CJ, O’Sullivan MG, Keohane C. Epithelioid hemangioendothelioma of the spine. Report of two cases. J Neurosurg Spine. 2005;3(5):393-9. doi:10.3171/spi.2005.3.5.0393. [PubMed: 16302636].

14. Yadav R, Roy M, Kumar T. Multifocal osseous epithelioid hemangioendothelioma involving extremities and spine with visceral metastasis. Indian J Med Paediatr Oncol.2018;39(1):103. doi:10.4103/ijmpo.ijmpo_31_16.

15. Bolke E, Gripp S, Peiper M, Budach W, Schwarz A, Orth K, et al. Multifocal epithelioid hemangioendothelioma: Case report of a clinical chamaeleon. EurJ Med Res. 2006;11(11):462-6. [PubMed: 17182357].

16. Abrahams TG, Bula W, Jones M. Epithelioid hemangioendothelioma of bone. A report of two cases and review of the literature. Skeletal Radiol. 1992;21(8):509-13. doi: 10.1007/bf00195232. [PubMed: 1465643].

17. Brennan JW, Midha R, Ang LC, Perez-Ordonez B. Epithelioid hemangioendothelioma of the spine presenting as cervical myelopathy: Case report. Neurosurgery. 2001;48(5):1166-9. doi: 10.1097/00006123-200105000-00045. [PubMed: 11334287].

18. Matamalas A, Gargallo A, Porcel JA, Garcia de Frutos A, Pellise F. Cervical spine epithelioid hemangioendothelioma: Case report. Eur Rev Med Pharmacol Sci. 2014;18(1 Suppl):72-5. [PubMed: 24825046].

19. Kopniczky Z, Tsimpas A, Lawson DD, King A, Pohl U, Pollock JR, et al. Epithelioid hemangioendothelioma of the spine: Report of two cases and review of the literature. BrJ Neurosurg. 2008;22(6):793-7. doi: 10.1080/02688690802158694. [PubMed: 19085365].

20. Ellis TS, Schwartz A, Starr JK, Riedel CJ. Epithelioid hemangioendothelioma of the lumbar vertebral column: Case report and review of literature. Neurosurgery. 1996;38(2):402-7. doi:10.1097/00006123-199602000-00036. [PubMed: 8869073].

21. Lee S. Hemangioendothelial sarcoma of the sacrum: CT findings. Comput Radiol.1986;10(1):51-3. [PubMed: 3956190].

22. Sebastian AS, Adair MJ, Morris JM, Khan MH, Arndt CA, Nassr A. Minimally invasive treatment of a painful osteolytic lumbar lesion secondary to epithelioid hemangioendothelioma. Global Spine J. 2015;5(2):135-9. doi:10.1055/s-0034-1387198. [PubMed: 25844287]. [PubMed Central: PMC4369204].

23. Christodoulou A, Symeonidis PD, Kapoutsis D, Iordanidis F. Primary epithelioid hemangioendothelioma of the lumbar spine. Spine J. 2008;8(2):38590. doi: 10.1016/j.spinee.2006.10.016. [PubMed: 18299105].

24. Ofluoglu AE, Aydogan M, Erdogan U, Ofluoglu O. Epithelioid hemangioendothelioma of the craniocervical junction; case report and review. Acta Orthop Traumatol Turc. 2017;51(4):342-6. doi:10.1016/j.aott.2017.03.012. [PubMed: 28478911]. [PubMed Central: PMC6197577].

25. Flucke U, Vogels RJ, de Saint Aubain Somerhausen N, Creytens DH, Riedl RG, van Gorp JM, et al. Epithelioid Hemangioendothelioma: Clinicopathologic, immunhistochemical, and molecular genetic analysis of 39 cases. Diagn Pathol. 2014;9:131. doi: 10.1186/1746-1596-9-131. [PubMed: 24986479]. [PubMed Central: PMC4100035].

26. Mendlick MR, Nelson M, Pickering D, Johansson SL, Seemayer TA, Neff JR, et al. Translocation t(1;3)(p36.3;q25) is a nonrandom aberration in epithelioid hemangioendothelioma. Am J Surg Pathol. 2001;25(5):684-7. doi:10.1097/00000478-200105000-00019. [PubMed: 11342784].

27. Antonescu CR, Le Loarer F, Mosquera JM, Sboner A, Zhang L, Chen CL, et al. Novel YAP1-TFE3 fusion defines a distinct subset of epithelioid hemangioendothelioma. Genes, Chromosomes Cancer. 2013;52(8):775-84. doi: 10.1002/gcc.22073. [PubMed: 23737213]. [PubMed Central: PMC4089994].

28. Reis C, Carneiro E, Fonseca J, Pereira P, Vaz R, Pinto R, et al. Epithelioid hemangioendothelioma and multiple thoraco-lumbar lateral meningoceles: two rare pathological entities in a patient with NF-1. Neuroradiology. 2005;47(2):165-9. doi:10.1007/s00234-004-1321-0. [PubMed:15688204].

29. Munier O, Muckensturm B, Fesneau M, Wachter T. [Epithelioid hemangioendothelioma of the spine: A case report]. Cancer Radiother. 2017;21(3):222-5. doi: 10.1016/j.canrad.2016.11.006. [PubMed: 28478891].

30. Cohen JE, Lylyk P, Ceratto R, Kaplan L, Umanskyt F, Gomori JM. Percutaneous vertebroplasty: Technique and results in 192 procedures. Neurol Res. 2004;26(1):41-9. doi: 10.1179/016164104773026516. [PubMed: 14977056]. 